Bull. Austral. Math. Soc.

Vol. 39 (1988) [439-441]

\title{
LOCAL BOUNDEDNESS OF MONOTONE OPERATORS UNDER MINIMAL HYPOTHESES
}

\author{
JoN BORWEIN AND Simon FitzPatrick
}

\begin{abstract}
We give a short proof the local boundedness of a monotone operator as an easy consequence of the continuity of an associated convex function.
\end{abstract}

Let $X$ be a real normed space with normed dual $X^{*}$. Recall that a set-valued mapping $T: X \rightarrow X^{*}$ is monotone if

$$
\left\langle x^{*}-y^{*}, x-y\right\rangle \geqslant 0
$$

for any pairs $x^{*} \in T(x), y^{*} \in T(y)$. The domain of $T, D(T)$, is defined by $D(T):=$ $\{x \in X: T(x) \neq \emptyset\}$. The mapping is locally bounded at $x \in D(T)$ if for some $\varepsilon>0$ the set $T(\{y \in X:\|y-x\|<\varepsilon\})$ is bounded. For any set $S$ in $X$, the cone generated by $S$ at $x$ is $S_{x}=\{y \in X \mid y=t(s-x), s \in S, t>0\}$. We will (a little non-standardly) call a point $x$ of $S$ an absorbing point of $S$ if $S_{x}=X$. Clearly every interior point is an absorbing point. If $S$ is convex then absorbing points and core points coincide.

A function $f: X \rightarrow]-\infty, \infty]$ is convex if epi $(f)=\{(x, r) \in X \times R \mid f(x) \leqslant r\}$ is a convex set, and lower semicontinuous if $\{x \in X \mid f(x) \leqslant r\}$ is closed for each real $r$. [Here $X$ may be any real topological vector space.] The essential domain of $f$ is $\operatorname{dom}(f)=\{x \in X: f(x)<\infty\}$. Finally, recall that a real locally convex space $X$ is barrelled if each closed convex set $C$ in $X$ for which 0 is an absorbing point [that is $C_{0}=X$ ] is a neighbourhood of zero. It follows from the Baire category theorem that every Banach space is barrelled, as indeed is every Baire normed space. Moreover, a Banach space may possess many non-Baire but barrelled normed subspaces including some dense hyperplanes [1]. The following result is basic to much convex analysis [2]. We include a proof of a somewhat more general version.

TheOREM 1. Let $X$ be a barrelled locally convex space. Let $f: X \rightarrow]-\infty, \infty$ ] be convex and lower semicontinuous and suppose that $x$ is an absorbing point of $\operatorname{dom}(f)$. Then $f$ is continuous at $\boldsymbol{x}$.

Proof: We may assume by translation that $f(0)=0$ and that 0 is an absorbing point of $\operatorname{dom}(f)$. Let $\varepsilon>0$ be given and set $C=\{x \in X \mid f(x) \leqslant \varepsilon\}$. Since $f$ is

Received 15 August 1988

Copyright Clearance Centre, Inc. Serial-fee code: 0004-9729/88 \$A2.00+0.00. 
convex and lower semicontinuous, $C$ is convex and closed. We verify that $U=C \cap-C$ is a symmetric neighbourhood of zero. As $X$ is barrelled it suffices to show that 0 is an absorbing point of $C$. Let $x \in X$. By hypothesis $f(r x)<\infty$ for some $r>0$. Select $\lambda>0$ such that $\lambda f(r x)<\varepsilon$ and $\lambda<1$. Then $\lambda r x$ lies in $C$ because

$$
f(\lambda r x) \leqslant \lambda f(r x)+(1-\lambda) f(0)=\lambda f(r x)<\varepsilon .
$$

Finally, since $f(-x)+f(x) \geqslant 0$, we have $|f(x)-f(0)| \leqslant \varepsilon$ for all $x$ in $U$.

We may now establish our main result.

Theorem 2. Let $X$ be a barrelled normed space. Let $T: X \rightarrow X^{*}$ be monotone and suppose that $x$ is an absorbing point of $D(T)$. Then $T$ is locally bounded at $x$.

Proof: We may assume by translation that $0 \in T(0)$ and that 0 is an absorbing point of $D(T)$ (which need not be convex). Consider the function $f: X \rightarrow]-\infty, \infty$ ] given by

$$
f(x)=\sup \left\{\left\langle y^{*}, x-y\right\rangle: y^{*} \in T(y),\|y\| \leqslant 1\right\} .
$$

Indeed, $f$ is always nonnegative since $0 \in T(0)$. Then $f$ is convex and lower semicontinuous as a supremum of continuous affine functionals. Let $x \in X$. As 0 is an absorbing point of $D(T)$, we can find $t>0$ with $T(t x) \neq \emptyset$. Choose any $u^{*}(t) \in T(t x)$. For each $y^{*} \in T(y)$

$$
\left\langle y^{*}, t x-y\right\rangle \leqslant\left\langle u^{*}(t), t x-y\right\rangle
$$

because $T$ is monotone. Since $0 \in T(0)$ this also shows $f(0)=0$. Hence

$$
f(t x) \leqslant \sup \left\{\left\langle u^{*}(t), t x-y\right\rangle:\|y\| \leqslant 1\right\}<\infty .
$$

This shows that 0 is an absorbing point of $\operatorname{dom}(f)$. Thus Theorem 1 ensures that for some $\delta>0, f(x) \leqslant 1$ whenever $\|x\| \leqslant 2 \delta<1$.

Equivalently, if $y^{*} \in T(y),\|y\| \leqslant 1,\|x\| \leqslant 2 \delta$, then

$$
\left\langle y^{*}, x\right\rangle \leqslant\left\langle y^{*}, y\right\rangle+1 \text {. }
$$

Hence if $y^{*} \in T(y)$ with $\|y\| \leqslant \delta$, then

$$
2 \delta\left\|y^{*}\right\|=\sup \left\{\left\langle y^{*}, x\right\rangle:\|x\| \leqslant 2 \delta\right\} \leqslant\left\|y^{*}\right\|\|y\|+1 \leqslant \delta\left\|y^{*}\right\|+1
$$

and $\left\|y^{*}\right\| \leqslant 1 / \delta$. This establishes that $T$ is locally bounded at 0 .

Remarks. 1. The usual result is established under the stronger assumptions that $x$ is an interior point of $D(T)$, and that $X$ is Banach or Baire $[4,3]$. In our setting this is not presumed. For instance, $D=\left\{(x, y): x^{2}+y^{2}=2|x|\right\} \cup\{(0, y):|y|=1\}$ has 0 as an absorbing point and so is a possible domain in $\mathbf{R}^{2}$.

2. We could establish directly that $\{x \mid f(x) \leqslant 1\}$ is a barrel and so omit Theorem 1. This would also omit the pleasant parallel between the statements of the two results.

3. This technique also works of $x \in \operatorname{core} \operatorname{conv} D(T)$, as in [4]. 


\section{REFERENCES}

[1] J.M. Borwein and D.A. Tingley, 'On supportless convex sets', Proc. Amer. Math. Soc. 04 (1985), 471-476.

[2] R.B. Holnes, Geometric Functional Analysis and Applications (Springer-Verlag, New York, 1975).

[3] R.R. Phelps, Convex functions, Monotone Operators and Differentiability, Lecture Notes in Mathematics (Springer-Verlag, University of Washington, 1989). (to appear) .

[4] R.T. Rockafellar, 'Local boundedness of nonlinear monotone operators', Mitchigan Math. J. 16 (1969), 397-407.

Department of Mathematics Statistics and Computer Science

Dalhousie University

Halifax NS B3H 3J5

Canada
Department of Mathematics and Statistics University of Auckland Private Bag

Auckland

New Zealand 